Article

\title{
Antibacterial Activity and Biodegradation of Cellulose Fiber Blends with Incorporated $\mathrm{ZnO}$
}

\author{
Domen Malis ${ }^{1}$, Barbka Jeršek ${ }^{1}\left[\right.$, Brigita Tomšič ${ }^{2}$, Danaja Štular ${ }^{2}{ }^{(0}$, Barbara Golja ${ }^{2}$, \\ Gregor Kapun ${ }^{3}$ iD and Barbara Simončič ${ }^{2, *}$ iD \\ 1 Biotechnical Faculty, University of Ljubljana, Jamnikarjeva 101, 1000 Ljubljana, Slovenia; \\ malis.domen@gmail.com (D.M.); Barbka.Jersek@bf.uni-lj.si (B.J.) \\ 2 Faculty of natural Sciences and Engineering, University of Ljubljana, Aškerčeva 12, 1000 Ljubljana, Slovenia; \\ brigita.tomsic@ntf.uni-lj.si (B.T.); danaja.stular@ntf.uni-lj.si (D.Š.); barbara.golja@ntf.uni-lj.si (B.G.) \\ 3 National Institute of Chemistry, Hajdrihova 16, 1000 Ljubljana, Slovenia; gregor.kapun@ki.si \\ * Correspondence: barbara.simoncic@ntf.uni-lj.si
}

Received: 17 September 2019; Accepted: 16 October 2019; Published: 17 October 2019

check for updates

\begin{abstract}
This research aimed to study the influence of lyocell with incorporated ZnO (CLY) for antibacterial activity and biodegradation of fiber blends composed of viscose (CV), flax (LI), and CLY. Fiber blended samples with an increased weight fraction of CLY fibers were composed, and single CLY, CV and LI fibers were also used for comparison. Antibacterial activity was determined for the Gram-negative Escherichia coli and the Gram-positive Staphylococcus aureus bacteria. The biodegradation of fiber blends was investigated by the soil burial test. The results show that the single CLY fibers exhibited high antimicrobial activity against both E. coli and S. aureus bacteria and that the presence of LI fibers in the blended samples did not significantly affect antibacterial activity against $E$. coli, but drastically decreased the antibacterial activity against $S$. aureus. LI fibers strongly promoted the growth of $S$. aureus and, consequently, impaired the antimicrobial performance of $\mathrm{ZnO}$ against this bacterium. The presence of CLY fibers slowed down, but did not prevent, the biodegradation process of the fiber blends, even at the highest $\mathrm{ZnO}$ concentration. The soil that was in contact with the fiber blended samples during their burial was not contaminated to such an extent as to affect the growth of sprouts, confirming the sustainability of the fiber blends.
\end{abstract}

Keywords: fiber blends; cellulose fibers; $\mathrm{ZnO}$; antibacterial activity; biodegradation; soil contamination

\section{Introduction}

Antimicrobial activity is one of the most important functional properties of cellulose fibers since these fibers are very easily attacked by both bacteria and fungi [1-4]. Excessive growth of microorganisms on the fibers can cause health and hygiene problems for the textile wearer, on the one hand, as well as lower the value of the textile product when in use, on the other [2]. However, when fabricating antimicrobial cellulose fibers, a greater risk appears to be that the incorporation of an antimicrobial agent may destroy the fiber's biodegradability, which represents a prominent characteristic and significant advantage of cellulose fibers from an ecological point of view. Namely, cellulose fibers are known as environmentally-friendly fibers because they are readily degraded by soil microorganisms [5]. Therefore, the creation of antimicrobial activity while maintaining the fiber's biodegradability remains a major challenge, which requires the careful selection of an antimicrobial agent and its concentration.

Among antimicrobial agents, $\mathrm{ZnO}$ has attracted a lot of attention due to the general perception that it is a safe, environmentally friendly, and biocompatible substance [6-8]. The antimicrobial activity 
of $\mathrm{ZnO}$ is directly related to its photocatalytic performance under $\mathrm{UV}$ irradiation, in which reactive oxygen species (ROS) are generated [9-13]. It is assumed that the generation of $\mathrm{H}_{2} \mathrm{O}_{2}$ and $\bullet \mathrm{OH}$, in particular, is crucial for the efficiency of $\mathrm{ZnO}$ as an antimicrobial agent. In addition to $\mathrm{ROS}, \mathrm{Zn}^{2+}$ cations released from $\mathrm{ZnO}$ dissolution as well as $\mathrm{ZnO}$ nanoparticles (NPs) contribute to the antimicrobial activity. It is believed that ROS can easily incorporate into the bacterial cell membrane, causing the disruption of cellular components via oxidative stress. $\mathrm{Zn}^{2+}$ cations can penetrate the bacterial cell, where they inhibit its action. If $\mathrm{ZnO}$ is present in the form of NPs, the direct contact of ZnO NPs with the bacterial cell wall results in the destruction of cell integrity. Accordingly, the mechanism of the antimicrobial activity of $\mathrm{ZnO}$ is strongly influenced by $\mathrm{ZnO}$ particle size and crystal structure as well as the environmental conditions. It is worth noting that under dark conditions, which apply for the biodegradation process, the antimicrobial activity of $\mathrm{ZnO}$ is assumed to be mainly attributed to $\mathrm{Zn}^{2+}$ cations and $\mathrm{ZnO}$ NPs and less related to ROS, since the generation of these species is conditioned by UV irradiation [13].

Our research aims to investigate the influence of $\mathrm{ZnO}$ on the antibacterial activity of cellulose fibers as well as their biodegradation. For this purpose, lyocell with incorporated $\mathrm{ZnO}$ (CLY), viscose $(\mathrm{CV})$, and flax (LI) fibers were used and the concentration of the $\mathrm{ZnO}$ was varied with the concentration of the CLY in the fiber blend. To increase the concentration of $\mathrm{ZnO}$ in the samples, the proportion of CLY fibers was increased by decreasing the proportion of CV at a constant content of LI in the blends. The reason for using these fiber blends was that CV/LI blends are very popular in the manufacturing of summer clothing, for which antimicrobial protection is of great importance. The antibacterial activity of samples was estimated against Escherichia coli as the Gram-negative and Staphylococcus aureus as the Gram-positive bacterium. An important goal of our research was also to determine the influence of LI fibers on the antimicrobial properties of fiber blends. Namely, according to the literature [14-16], LI fibers have a reputation for being intrinsically antibacterial. The biodegradation of the samples was estimated by the soil burial test. It was hypothesized that the antimicrobial activity of $\mathrm{ZnO}$ in the soil would be impaired, which would beneficially affect the biodegradation process of the fiber blends.

\section{Materials and Methods}

\subsection{Materials}

Three types of cellulose fibers were used, i.e., antimicrobial lyocell with incorporated $\mathrm{ZnO}$ (CLY, Smart Fiber AG, Rudolstadt, Germany), viscose (CV, Lenzing, Austria), and flax (LI, Liniere de Saint Martin, France). In CLY production, $16 \%$ of $\mathrm{ZnO}$ was embedded into the fibers by the solvent spinning technique.

For the antibacterial tests, seven samples of three-component CLY/CV/LI fiber blends with a mass of $1.0 \pm 0.1 \mathrm{~g}$ were prepared with an increased weight fraction of CLY fibers $(0 \%, 15 \%, 20 \%$, $25 \%, 30 \%, 55 \%$, and $70 \%$ ) along with the appropriate decrease in the weight fraction of CV fibers and a constant amount of LI fibers $(30 \%)$. Furthermore, one sample was prepared of a two-component fiber blend composed of CLY and CV. The samples were prepared by weighing the individual fibers and assembling them into blends. Single CLY, CV, and LI fiber samples of the same mass were also prepared for the investigation. The sample codes and their compositions are summarized in Table 1.

For the soil burial test, five samples named K (control), CLY(25), CLY(70), CLY(100), CV(100), and $\mathrm{LI}(100)$ were selected. The mass of the samples was $15.0 \pm 0.1 \mathrm{~g}$. The samples were prepared by weighing the individual fibers and mixing them together with a stirrer (Shirley trash analyzer, Ikon Industries, India) to achieve a homogeneous mixture. The nonwoven fabric samples were put into models with dimensions of $24 \mathrm{~cm} \times 21 \mathrm{~cm}$ and clamped between wooden plates with the help of clamps. After 7 days, the clamps were removed, and the compressed samples were wrapped in plastic net. 
Table 1. Sample codes and blend ratios.

\begin{tabular}{cccc}
\hline Sample Code & $w_{\text {CLY }}(\mathbf{\%})$ & $w_{\text {CV }}(\mathbf{\%})$ & $w_{\text {LI }}(\mathbf{\%})$ \\
\hline K & 0 & 70 & 30 \\
CLY(15) & 15 & 55 & 30 \\
CLY(20) & 20 & 50 & 30 \\
CLY(25) & 25 & 45 & 30 \\
CLY(30) & 30 & 40 & 30 \\
CLY(55) & 55 & 15 & 30 \\
CLY(70) & 70 & 0 & 30 \\
CLY(30)-a & 30 & 70 & 0 \\
CLY(100) & 100 & 0 & 0 \\
CV(100) & 0 & 100 & 0 \\
LI(100) & 0 & 0 & 100 \\
\hline
\end{tabular}

Legend: CLY: lyocell with incorporated $\mathrm{ZnO}, \mathrm{CV}$ : viscose, and LI: flax.

\subsection{Methods}

\subsubsection{Inductively Coupled Plasma Mass Spectroscopy}

The amount of $\mathrm{ZnO}$ in the CLY(100) sample was determined by inductively coupled plasma mass spectroscopy (ICP-MS) using a Perkin Elmer SCIED Elan DRC spectrophotometer. A sample of $0.5 \mathrm{~g}$ was prepared in the Milestone microwave system using acid decomposition with $60 \%$ nitric acid and $30 \%$ hydrogen peroxide. Three measurements were carried out, and the $\mathrm{ZnO}$ concentrations are reported as the mean values and standard deviation (SD).

\subsubsection{Scanning Electron Microscopy with Energy-Dispersive X-ray Spectroscopy}

Scanning electron microscopy (SEM) was performed on a JEOL JSM 6060 LV SEM instrument operating with a primary electron beam of $10 \mathrm{kV}$ accelerating voltage and a working distance of $17 \mathrm{~mm}$. The samples were coated with a thin layer of gold before observation to increase the clarity of the images. SEM analysis was also performed using an SEM Ultra+ microscope (Zeiss, Oberkochen, Germany) equipped with an energy-dispersive X-ray spectrometer (EDS) X-Max 50 (Oxford, UK). The samples were coated with a thin layer of platinum before observation. Sample inspection was carried out with a low acceleration primary electron beam (1 kV at $40 \mathrm{pA})$ with the detection of secondary electrons.

\subsubsection{Fourier Transform-Infrared (FT-IR) Spectroscopy}

Fourier transform-infrared (FT-IR) spectra were obtained on a Spectrum GX I spectrophotometer (Perkin Elmer, Great Britain) equipped with an attenuated total reflection (ATR) cell and a diamond crystal $(\mathrm{n}=2.0)$. The spectra were recorded over a range of 4000 to $600 \mathrm{~cm}^{-1}$ using 32 scans at a resolution of $4 \mathrm{~cm}^{-1}$.

\subsubsection{Antibacterial Activity}

The antibacterial activity of samples was estimated for the Gram-negative bacterium Escherichia coli ATCC 11229 and the Gram-positive bacterium Staphylococcus aureus ATCC 25923 according to the ASTM E 2149-01 standard method [17]. A fabric sample of $1 \mathrm{~g}$ was immersed in $50 \mathrm{~mL}$ of an NB (nutrient broth, Oxoid, CM0067, Basingstoke, UK) medium of known bacterial concentration in a flask, which was then shaken using a wrist-action shaker. To provide efficient leaching of ZnO from the CLY fibers into the NB culture, $2 \mathrm{~h}$ of contact time was selected. Afterwards, viable bacteria were quantified with plate count method using NA (nutrient agar, Biolife, 4018102, Milano, Italy) and incubation of NA plates at $37^{\circ} \mathrm{C}$ for $24 \mathrm{~h}$. Two parallel assessments were performed for each fabric sample. The number of colonies was counted, and if the duplicate counts of any sample did not agree within 15\%, we 
discarded that sample and repeated the test. Afterwards, the number of colonies was converted to the number of bacteria as colony-forming units per $\mathrm{mL}(\mathrm{CFU} / \mathrm{mL})$. The reduction in the number of bacteria, $R$, was calculated as follows:

$$
R=\frac{(B-A)}{B} \times 100(\%)
$$

where $R$ is the bacterial reduction, $A$ is the number of bacteria in a flask containing a sample with incorporated antimicrobial CLY fibers after $2 \mathrm{~h}$ of contact time, and $B$ is the number of bacteria in a flask containing a reference sample without antimicrobial CLY fibers after $2 \mathrm{~h}$ of contact time. The $R$ value is reported as the mean value and standard error.

To determine the number of viable bacterial cells in the NB medium before and after the specified contact time, the number of bacteria at " 0 " contact time and after 2 and $24 \mathrm{~h}$ of contact with fibers was determined. To determine the number of adhered bacteria, the flasks containing the fibers in contact for $24 \mathrm{~h}$ were previously treated in the Sonis 10GT ultrasonic apparatus (Iskra PIO d.o.o., Slovenia) for $10 \mathrm{~min}(30 \mathrm{kHz}, 1100 \mathrm{~W})$ [18]. For comparison, the number of bacteria in the flask containing the inoculum only was determined after the specified contact time.

\subsubsection{Soil Burial Test}

The soil burial test was carried out in accordance with the ISO 11721-1:2001 and ISO 11721:2003 standards $[19,20]$. Following this standard procedure, the container was filled with commercial grade compost. The water content of the soil was $60 \pm 5 \%$ of its maximum moisture retention capacity. This was held constant during the experiment by spraying with water. The $\mathrm{pH}$ of the soil was between 4.0 and 7.5. Fabric samples were buried in the soil for periods of 10, 20, 30, and 50 days. After the defined incubation time, the samples were removed from the test soil, rinsed with running tap water and immersed in $70 \%$ ethanol for $30 \mathrm{~min}$ before air drying.

\subsubsection{Soil Contamination}

To investigate the potential impact of buried samples on the soil contamination, soil that was in direct contact with samples K, CLY(25), and CLY(70) during 50 days of burial was transferred into plastic pots. Soil that was not in contact with the fabric samples was used as a reference. Fifty seeds of Sinapis arvensis were planted into each pot, and the sprouts were counted after 14 days of growth. Four parallel assessments were performed for each sample, and the mean value and standard deviation were determined. The influence of soil on sprout growth was determined by analysis of variance (ANOVA, F test, $1 \%$ level of probability).

\section{Results and Discussion}

\subsection{Characterization of CLY Fibers}

The SEM images of CLY fibers in Figure 1a,b show the surface morphological properties as well as the distribution of $\mathrm{ZnO}$ particles incorporated into the fibers. It was observed that $\mathrm{ZnO}$ particles of irregular shapes and particle sizes ranging from 70 to $800 \mathrm{~nm}$ were evenly distributed on the CLY fibers. The EDS spectrum (Figure 1c) clearly revealed a characteristic peak at $1.03 \mathrm{keV}$, which was attributed to $\mathrm{Zn}$.

According to the ICP-MS analysis, the concentration of ZnO in the CLY(100) sample was $120 \pm 1 \mathrm{mg} / \mathrm{g}$. 

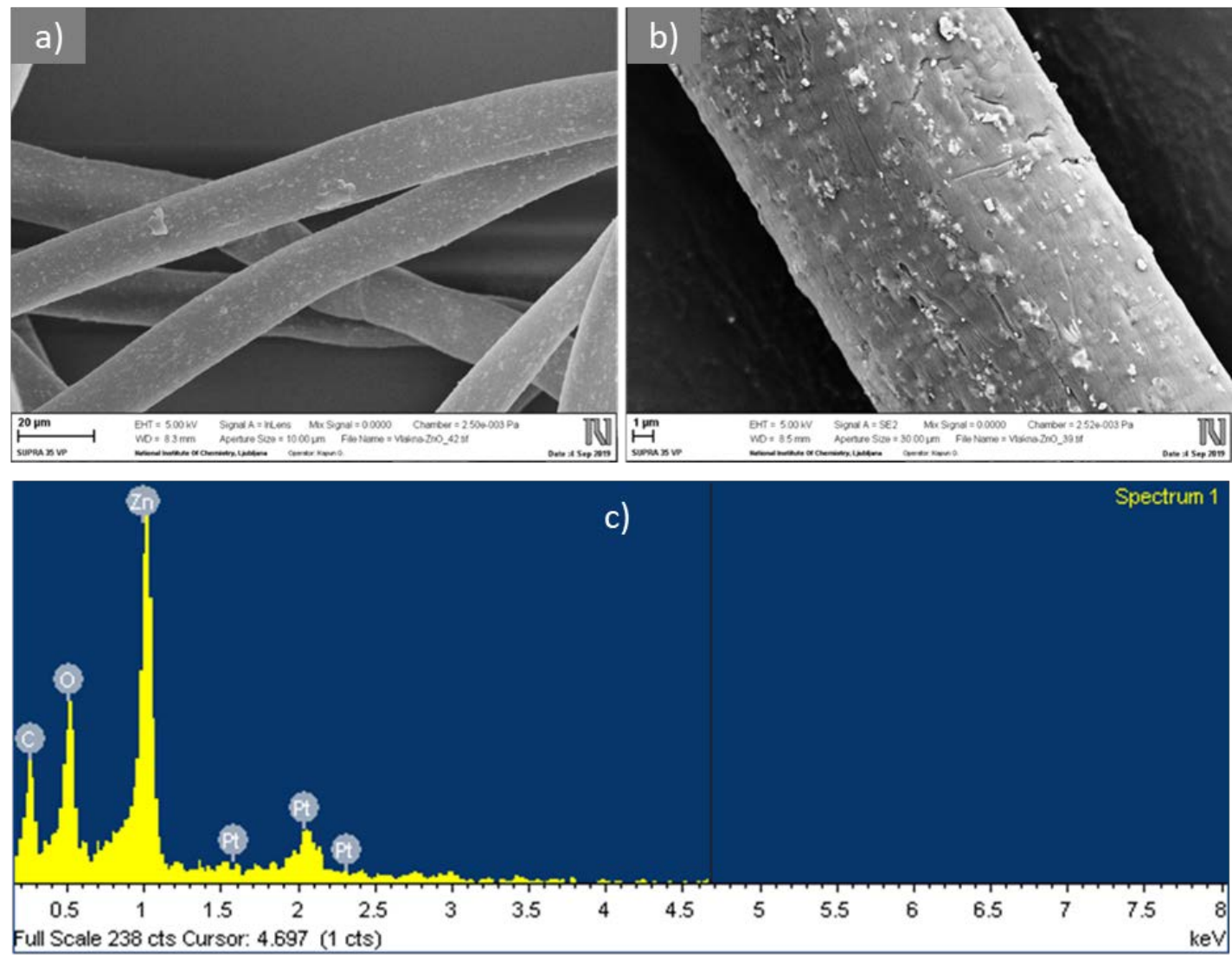

Figure 1. Scanning electron microscopy/Backscattered-Electron images (SEM/BSE) of CLY fibers at lower (a) and higher (b) magnification and energy-dispersive X-ray spectrometer (EDS) spectrum acquired from $\mathrm{ZnO}$ particles (c).

\subsection{Antibacterial Activity}

The results of the antibacterial activity are presented in Figure 2. It was observed that, irrespective of the content of the CLY component in the fiber blends, antimicrobial activity was more efficient against $E$. coli than against $S$. aureus. Sufficient antibacterial activity, with $R$ equal to $86 \%$, was obtained for the CLY(25) sample, and it slightly increased when the CLY content was increased from $25 \%$ to $70 \%$ in sample CLY(70). This suggests bacteriostatic activity against $E$. coli. On the other hand, low bacterial reduction against $S$. aureus was obtained for all studied samples irrespective of the CLY content, which was unexpected, as it is generally believed that $\mathrm{ZnO}$ is more effective against Gram-positive than against Gram-negative bacteria [21-24]. The results were not in accordance with those from the literature, where, despite higher sensitivity of $E$. coli than $S$. aureus to $\mathrm{ZnO}$, a significant antibacterial protection for both Gram-positive and Gram-negative bacteria was observed for different $\mathrm{ZnO}$-functionalized synthetic fibers $[25,26]$. Similarly, excellent bacterial reduction of E. coli and S. aureus was also reported for $\mathrm{ZnO} / \mathrm{Ag}$ composite functional polyester nonwoven fabric [27].

To investigate the reason for the results obtained in the case of the studied cellulose fiber blends, the antimicrobial activity of single CLY fibers (sample CLY(100)) as well as their blend with CV fibers were investigated and compared to the results obtained for the three-component CLY/CV/LI blended samples (Figure 3). As seen in Figure 3, single CLY fibers exhibited high antimicrobial activity against both $E$. coli and S. aureus bacteria, and the bacterial reduction against $S$. aureus was expectedly higher than that against $E$. coli. The bacterial reductions caused by CLY(30)-a sample composed of $30 \%$ CLY and $70 \%$ CV fibers-were very similar to those obtained for the single CLY fibers, indicating the excellent antimicrobial activity of $\mathrm{ZnO}$ even at lower CLY content. Surprisingly, the antibacterial activity against $S$. aureus drastically decreased when LI fibers were included into the fiber blends 
(samples CLY(30) and CLY(70)), regardless of the CLY weight fraction. According to this, it can be suggested that the LI fibers in the fiber blends represented a secondary source of nutrients, promoting the bacteria growth and, consequently, impairing the antimicrobial performance of $\mathrm{ZnO}$. These results are the opposite to those from the literature, in which antibacterial activity of flax fibers was achieved as a result of the action of phenolic compounds in lignin [14-16].

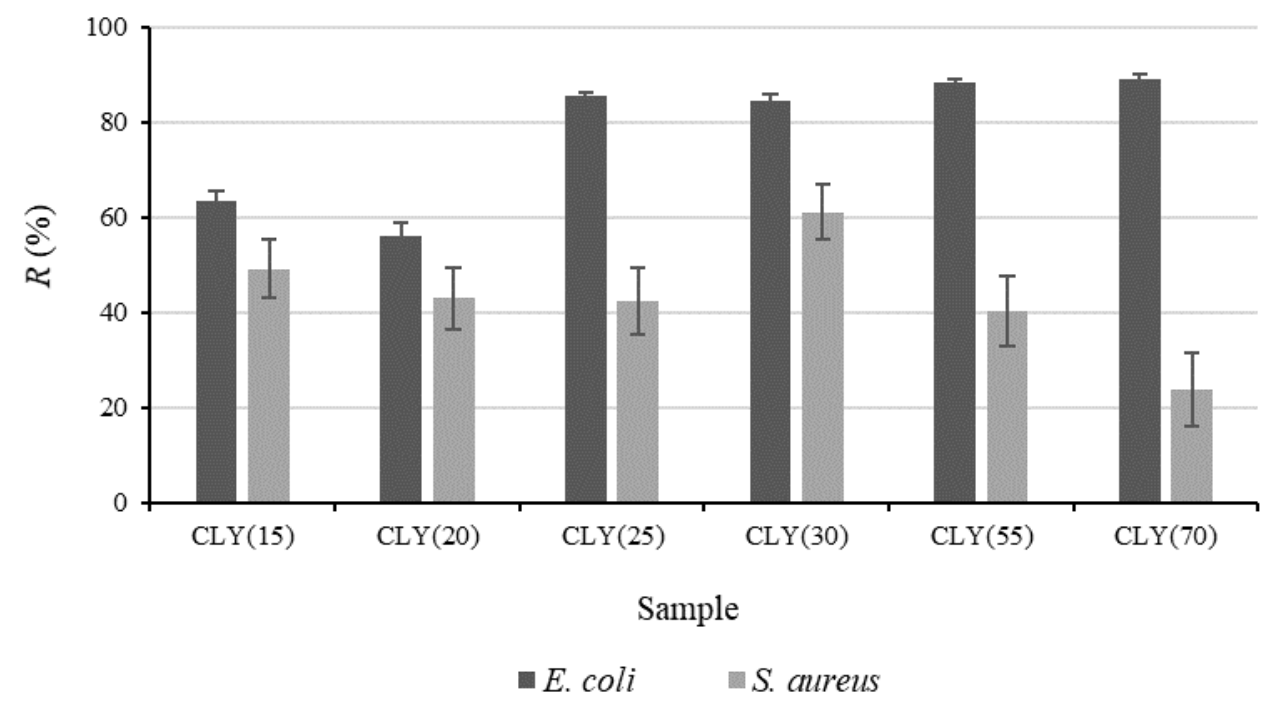

Figure 2. Reduction, $R$, of Escherichia coli and Staphylococcus aureus versus the mass fraction of CLY fibers, $w_{\mathrm{CLY}}$, in the fiber blend samples CLY(15) to CLY(70). The K sample was used as a reference.

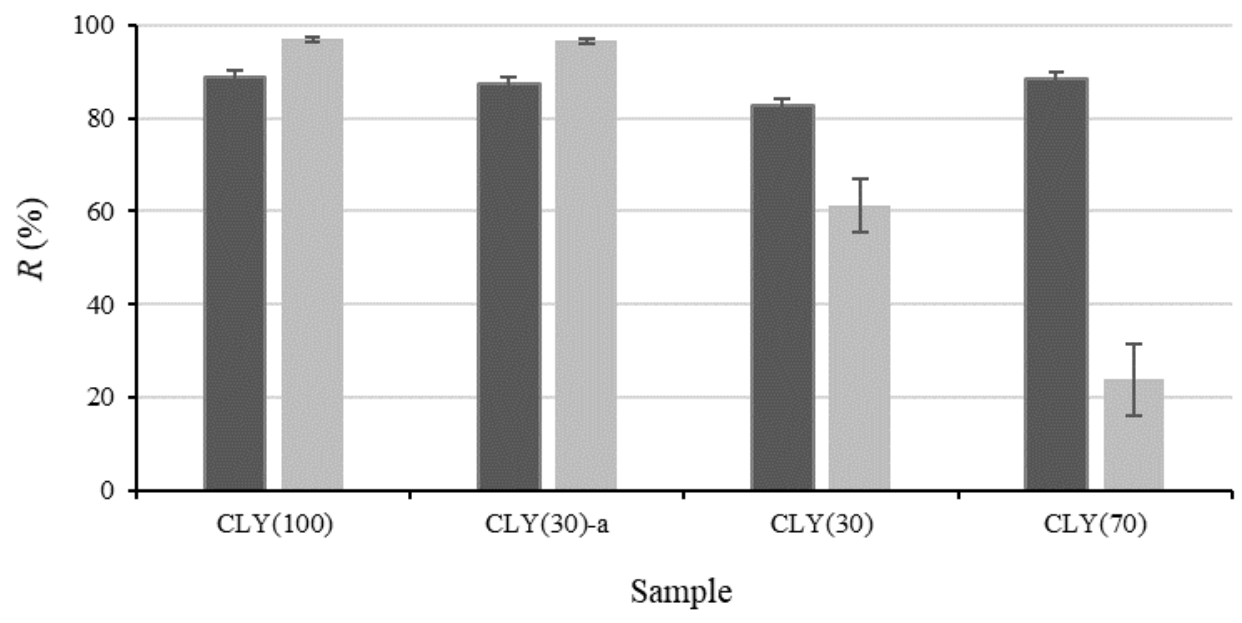

E. coli $\quad$ S. aureus

Figure 3. Reduction, $R$, of E. coli and S. aureus for single CLY fibers (sample CLY(100)) and the fiber blended samples. The K sample was used as a reference for the CLY(30) and CLY(70) samples and the CV(100) sample was used as a reference for the CLY(100) and CLY(30)-a samples.

To gain insight into our results, the differences between the numbers of viable bacterial cells after $2 \mathrm{~h}$ of incubation and at contact time " 0 ", without and in the presence of fiber blended samples, were determined for both bacteria, and the results are presented in Figure 4. It was found that the numbers of both bacteria increased after incubation in the inoculum without any sample and in the presence of sample K, which contained no CLY fibers. Compared to the inoculum itself, the presence of sample K provided much more favorable conditions for the growth of $S$. aureus than for that of $E$. coli. In spite of the fact that the replacement of CV fibers by antimicrobial CLY fibers in the CLY(55) and CLY(70) 
samples decreased the $\Delta N$ value for $S$. aureus compared to that obtained in inoculum alone, this could not prevent the bacterial growth during incubation (Figure $4 \mathrm{~b}$ ). Definitely, LI fibers strongly promoted the growth of $S$. aureus, and therefore the concentration of $\mathrm{ZnO}$ leached from CLY fibers was too low to provide sufficient antibacterial activity, even at $70 \%$ of CLY fibers in the sample. This phenomenon was not observed for $E$. coli, where the $\mathrm{ZnO}$ leached from CLY fibers significantly reduced the number of viable bacteria cells during incubation (Figure 4a).

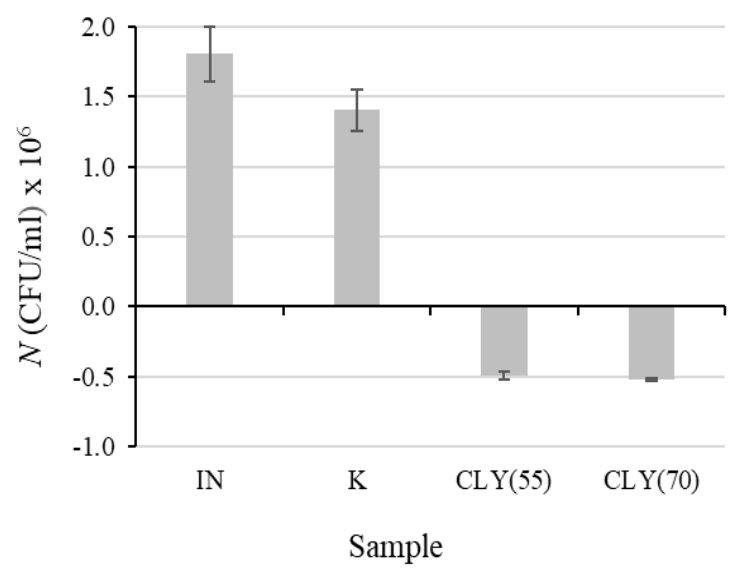

(a)

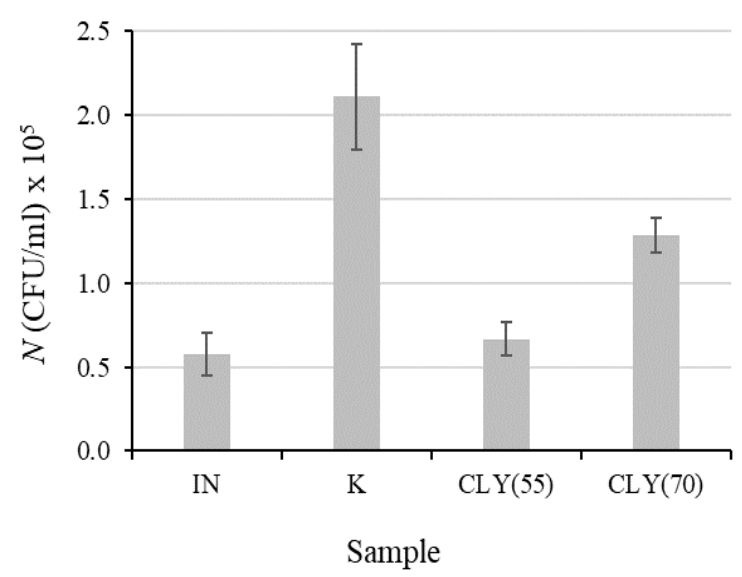

(b)

Figure 4. Difference between the numbers of viable bacteria cells, $\Delta N$, after and before $2 \mathrm{~h}$ of incubation for E. coli (a) and S. aureus (b) in inoculum (IN) alone and in the presence of samples K, CLY(55) or CLY(70). The numbers on the $x$ axis denote the number of the CLY sample.

To verify our findings, the bacterial growth in the presence of single LI fibers (sample LI(100)) was investigated, and the results are summarized in Table 2 and Figure 5. As seen in Table 2, the number of bacteria increased with increased incubation time for both bacteria. Whereas E. coli had no tendency for adhering onto LI fibers, a strong adhesion ability was noticed for S. aureus. SEM images of LI fibers after incubation revealed that only rare individual cells of $E$. coli were present on the fiber surface (Figure 5a), while cell clusters protected by biofilm (Figure $5 c, d$ ) were observed beside individual cells of $S$. aureus (Figure $5 b$ ) in many places, confirming the suitability of LI fibers as growth media. To determine the proper number of viable bacterial cells, fiber treatment with ultrasound was necessary. This should be taken into account if standard tests based on the colony count are used.

Table 2. Number of viable bacteria cells, $N$, for E. coli and $S$. aureus incubated in the presence of the $\mathrm{LI}(100)$ sample for different periods of time, $t$, as well as before and after $10 \mathrm{~min}$ of ultrasound treatment.

\begin{tabular}{ccccc}
\hline \multirow{2}{*}{ Bacterium } & $\boldsymbol{4} \mathbf{c} \mathbf{N}(\mathrm{CFU} / \mathbf{m L})$ \\
\cline { 2 - 5 } & $\boldsymbol{t}=\mathbf{2} \mathbf{h}$ & $\begin{array}{c}\boldsymbol{t}=\mathbf{2 4} \mathbf{h} \text {, before } \\
\text { Ultrasound }\end{array}$ & $\begin{array}{c}\boldsymbol{t}=\mathbf{2 4} \mathbf{h} \text {, after } \\
\text { Ultrasound }\end{array}$ \\
\hline E. coli & $6.58 \times 10^{5}$ & $2.30 \times 10^{6}$ & $7.75 \times 10^{7}$ & $7.29 \times 10^{7}$ \\
S. aureus & $2.63 \times 10^{5}$ & $3.32 \times 10^{5}$ & $3.90 \times 10^{4}$ & $1.96 \times 10^{7}$ \\
\hline
\end{tabular}



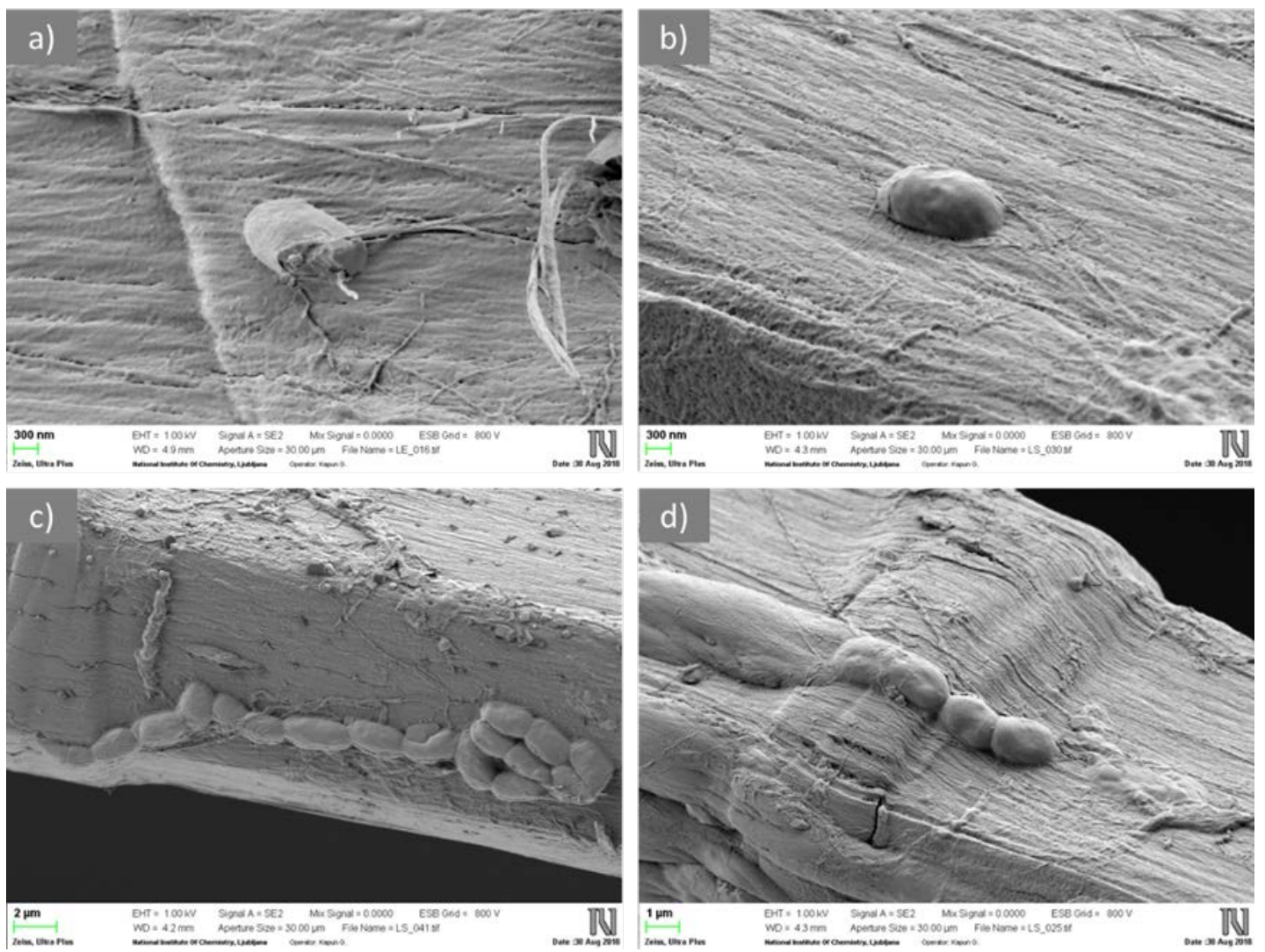

Figure 5. SEM images of E. coli (a) and S. aureus (b-d) on the surface of LI fibers.

\subsection{Biodegradation of Fiber Blends}

The influence of CLY fibers in the blended samples on their biodegradation was studied by a soil burial test. In this respect, the following three fiber blended samples were taken into consideration for various reasons: the CLY(25) sample, for which sufficient antibacterial activity was obtained for $E$. coli, the CLY(70) sample, which had the highest content of CLY fibers, and the K sample without CLY fibers as a control. Photos of the samples removed from the test soil after different incubation times are presented in Figure 6. Since the microorganisms in the soil caused fiber rotting, which was reflected in the samples' browning of the fiber surface, the intensity of the color change could represent a measure of the biodegradation rate. Furthermore, the progression of biodegradation was also reflected in the formation of holes in the samples followed by the tearing of samples into pieces.

A comparison of the photos revealed that the presence of ZnO in the CLY(25) and CLY(70) samples slowed down the biodegradation process compared to the K sample, but it did not prevent the fibers' biodegradation, even at the highest concentration in the CLY(70) sample. This finding indicated that the biodegradability of the studied samples was maintained. The reason for this could be explained by the fact that the antimicrobial activity of $\mathrm{ZnO}$ is photocatalytic, driven by the formation of reactive oxygen species under UV radiation, and that this mechanism of action was not present during the samples' burial [13]. At the same time, $\mathrm{ZnO}$ exhibits poor activity against fungi [28], which represent the majority of soil microorganisms. These results are similar to those obtained for $\mathrm{ZnO}$-coated cellulose fibers [29] but are very different from those obtained for the AgCl-functionalized cellulose fibers, where $\mathrm{Ag}$ cations that released from the fiber's surface exhibited high biocidal activity [30]. 

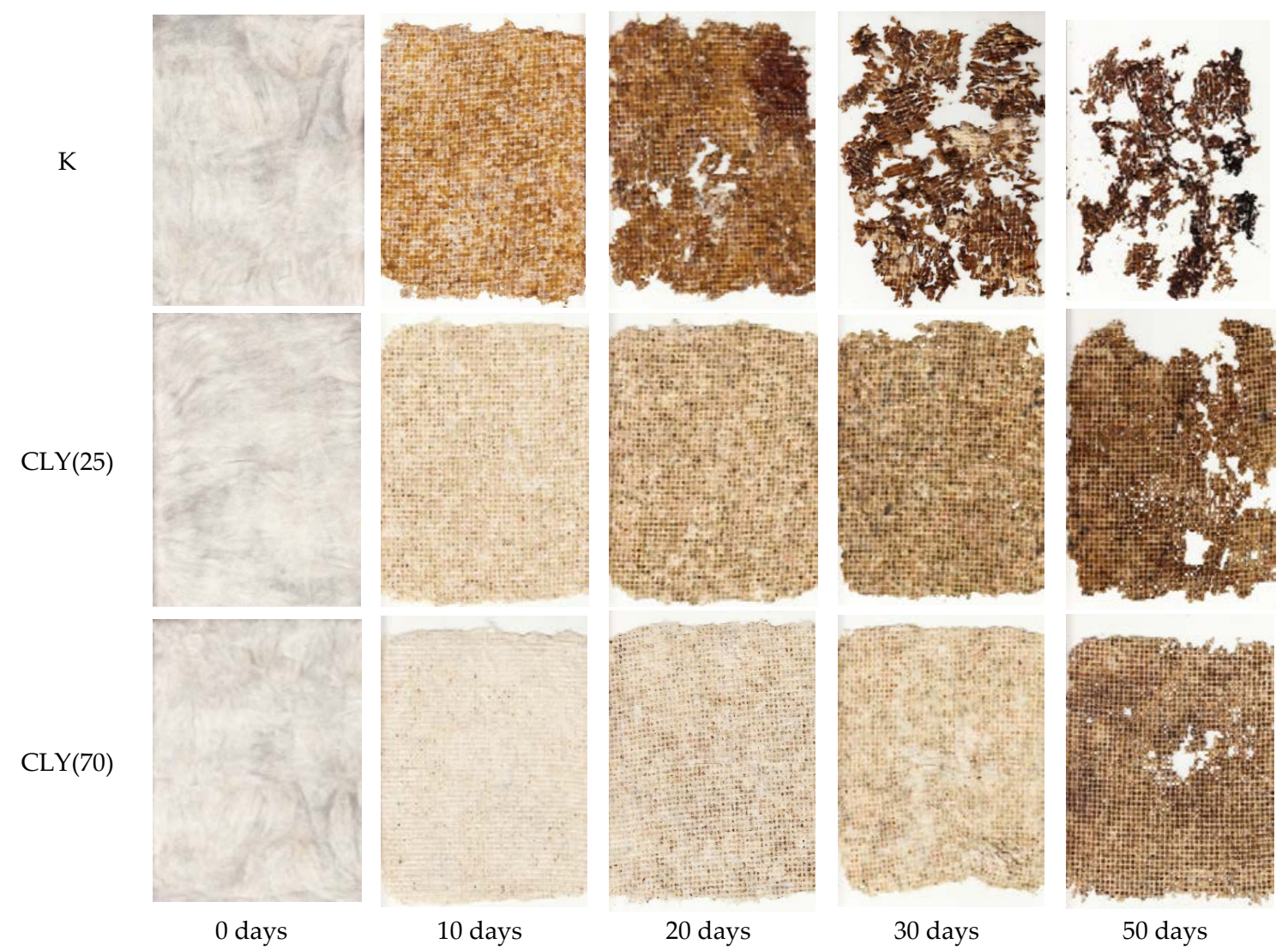

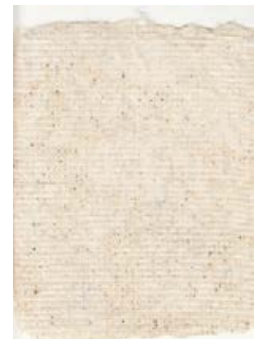

10 days

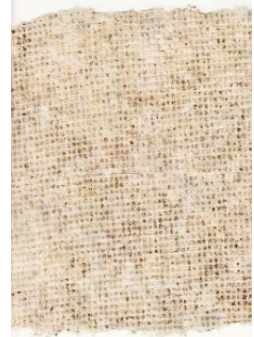

20 days

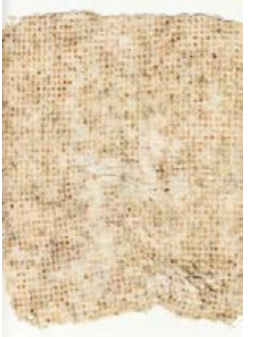

30 days

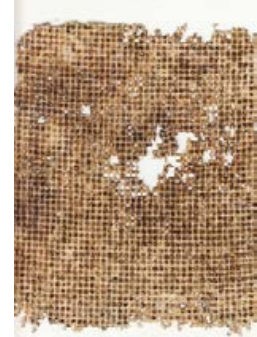

50 days

Figure 6. Photos of the K, CLY(25), and CLY(70) samples before (0 days) and after 10, 20, 30, and 50 days of soil burial.

The growth of microorganisms on the studied samples during biodegradation in soil was inspected by FT-IR spectroscopy (Figure 7). According to our previous study [31], important information about the progress of the biodegradation process can be obtained from the intensity of the bands at 1640 and $1548 \mathrm{~cm}^{-1}$, belonging to the amides I and II groups of secondary polyamides [32], resulting from the presence of protein produced by microbial growth on fibers.

It is seen from the ATR spectra of the CLY(25) sample (Figure 7) that the intensity of the bands at 1640 and $1548 \mathrm{~cm}^{-1}$ significantly increased with increased periods of soil burial. Furthermore, both bands were also clearly exposed in the spectrum of $\operatorname{CLY}(70)$ after 50 days of soil burial (Figure 8a), confirming the microbial growth on this fiber blend despite the high concentration of $\mathrm{ZnO}$. The progression of biodegradation was also reflected in the decreased intensities of bands in the $1200-900 \mathrm{~cm}^{-1}$ spectral region due to structural changes in the cellulose fingerprint. Simultaneously, a band at $1735 \mathrm{~cm}^{-1}$ due to $\mathrm{C}=\mathrm{O}$ stretching in aldehyde groups appeared as a result of cellulose chain degradation [32].

Figure 8 shows the results of the growth of Sinapis arvensis sprouts in soil, which was or was not in contact with the K, CLY(25), or CLY(70) samples during 50 days of burial. In none of the pots did all 50 seeds sprout (Figure $8 \mathrm{~b}$ ). This was also true for the seeds that were grown in the reference soil. Although a slightly lower mean value of sprout number was obtained for soil in contact with CLY(70) in comparison to the other samples, according to ANOVA, the soil did not have a significant influence on the sprout growth. This suggested that the rotting of samples and the leaching of $\mathrm{ZnO}$ from the samples during the soil burial did not significantly affect the growth conditions for the sprouts, which, consequently, confirmed the sustainability of the fiber blends. 


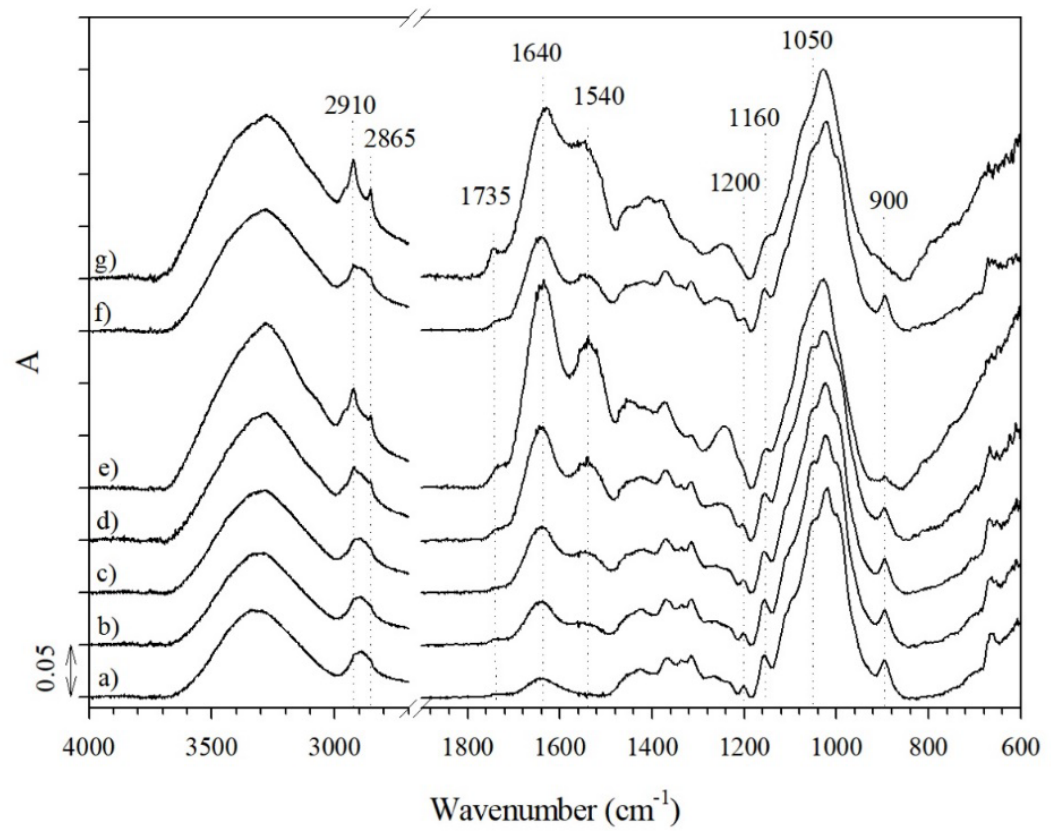

Figure 7. Attenuated total reflection (ATR) spectra of the CLY(25) sample before (a) and after 10 (b), 20 (c), 30 (d), and 50 (e) days of soil burial, as well as the CLY(70) (f) and K (g) samples after 50 days of soil burial.

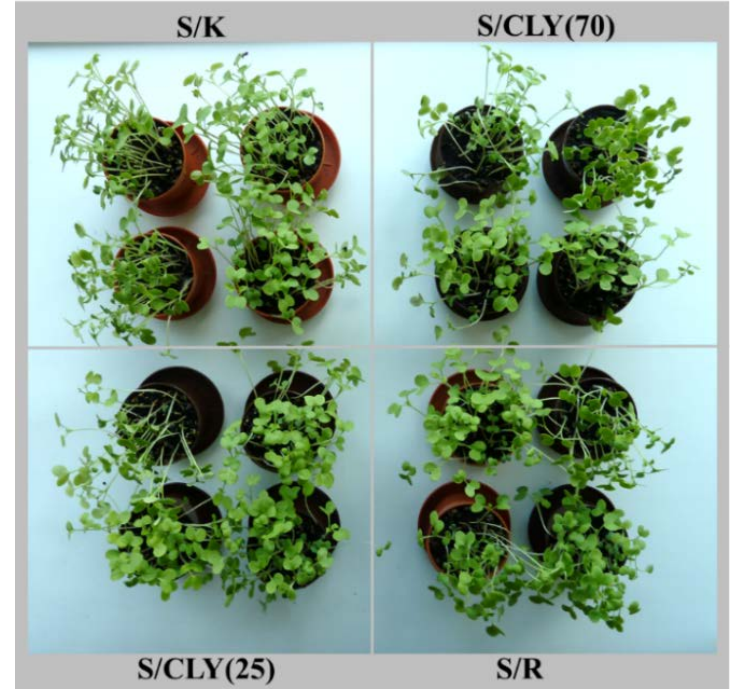

(a)

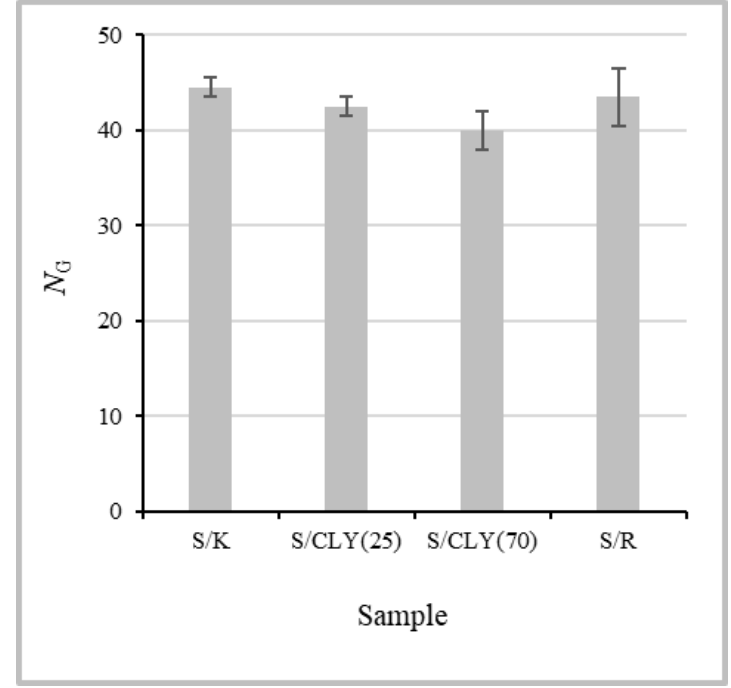

(b)

Figure 8. Growth of Sinapis arvensis sprouts in soil, which was in contact with samples K (S/K sample), CLY(25) (S/CLY(25) sample), and CLY(70) (S/CLY(70) sample) during 50 days of burial and in the reference soil (S/R sample) with no sample contact (a) and number, $N_{\mathrm{G}}$, of sprouts grown in the soil in 14 days (b).

\section{Conclusions}

This study highlights the influence of $\mathrm{ZnO}$ on the antibacterial activity and biodegradability of fiber blends composed of CLY, CV, and LI fibers of different blend ratios. The results show that the fiber composition in the samples strongly influenced their antibacterial activity. Whereas CLY fibers blended with CV exhibited high antibacterial activity against both E. coli and S. aureus, the presence of LI fibers in the three-component blends drastically hindered their antibacterial activity against $S$. aureus. Therefore, LI fibers did not exhibit antimicrobial properties, but, in contrast, they even promoted 
bacterial growth and, consequently, impaired the antimicrobial performance of $\mathrm{ZnO}$. This phenomenon was accompanied by high adhesion of $S$. aureus onto the LI fibers and the formation of a biofilm.

The presence of $\mathrm{ZnO}$ slowed down the biodegradation process of the fiber blends compared to the sample that did not include CLY fibers, but ZnO did not eliminate the fibers' biodegradation, even at the highest concentration. This was confirmed by the increased intensity of the absorption bands belonging to the amides I and II groups of secondary polyamides due to the protein portion, indicating the microbial growth on the fibers. Furthermore, the rotting of fiber blends and the leaching of $\mathrm{ZnO}$ from the samples during soil burial did not significantly contaminate the soil, proving the sustainability of the fiber blends.

Author Contributions: Conceptualization, D.M., B.J., and B.S.; Methodology, D.M., B.J., and B.S.; Investigation, D.M., D.Š., B.T., B.G., and G.K.; Validation, D.M., B.J., and B.S.; Writing-original draft, B.S. and D.M.; Writing-review, B.S., D.M., B.J., B.T., D.Š., B.G., and G.K.

Funding: This work was co-founded by the Slovenian Research Agency (Programs P2-0213 and P4-0116, Infrastructural Center RIC UL-NTF).

Acknowledgments: We thank Veronika Vrhunc and Franci Debelak, Litia Spinnery, for supplying the fibers and constructive discussion. We also thank Mateja Kokalj for her kind assistance.

Conflicts of Interest: The authors declare no conflict of interest.

\section{References}

1. Szostak-Kotowa, J. Biodeterioration of textiles. Int. Biodeterior. Biodegrad. 2004, 53, 165-170. [CrossRef]

2. Vigo, T.L. Protection of Textiles from Biological Attack. In Functional Finishes, Part A, Handbook of Fibre Science and Technology; Lewin, M., Sello, S.B., Eds.; Marcel Dekker, INC.: New York, NY, USA, 1983; Volume 2, pp. 367-426, ISBN 0-8247-1716-3.

3. Schindler, W.D.; Hauser, P.J. Antimicrobial Finishes. In Chemical Finishing of Textiles; Woodgead Publishing Limited: Camridge, UK, 2004; pp. 165-174. ISBN 1-85573-905-4.

4. Simončič, B.; Tomšič, B. Recent Concepts of Antimicrobial Textile Finishes. In Textile Finishing: Recent Developments and Future Trends; Mital, K.L., Bahners, T., Eds.; John Wiley \& Sons: Hoboken, NJ, USA, 2017; Part 1; pp. 3-68, ISBN 978-1-119-42676-9.

5. Park, C.H.; Kang, Y.K.; Im, S.S. Biodegradability of cellulose fabrics. J. Appl. Polym. Sci. 2004, 94, $248-253$. [CrossRef]

6. Hackenberg, S.; Scherzed, A.; Technau, A.; Froelich, K.; Hagen, R.; Kleinsasser, N. Functional responses of human adipose tissue-derived mesenchymal stem cells to metal oxide nanoparticles in vitro. J. Biomed. Nanotechnol. 2013, 9, 86-95. [CrossRef]

7. Reddy, A.R.N.; Srividya, L. Evaluation of in vitro cytotoxicity of zinc oxide (ZnO) nanoparticles using human cell lines. J. Toxicol. Risk Assess. 2018, 4. [CrossRef]

8. Verbič, A.; Šala, M.; Gorjanc, M. The influence of in situ synthesis parameters on the formation of $\mathrm{ZnO}$ nanoparticles and the UPF value of cotton fabric. Tekstilec 2018, 61, 280-288. [CrossRef]

9. Sirelkhatim, A.; Mahmud, S.; Seeni, A.; Kaus, N.H.M.; Ann, L.C.; Bakhori, S.K.M.; Hasan, H.; Mohamad, D. Review on Zinc Oxide Nanoparticles: Antibacterial Activity and Toxicity Mechanism. Nano-Micro Lett. 2015, 7, 219-242. [CrossRef]

10. Kumar, R.; Umar, A.; Kumar, G.; Nalwa, H.S. Antimicrobial properties of ZnO nanomaterials: A review. Ceram. Int. 2017, 43, 3940-3961. [CrossRef]

11. Siddiqi, K.S.; Rahman, A.U.; Husen, A. Properties of Zinc Oxide Nanoparticles and Their Activity Against Microbes. Nanoscale Res. Lett. 2018, 13, 141. [CrossRef]

12. Mishra, P.K.; Mishra, H.; Ekielski, A.; Talegaonkar, S.; Vaidya, B. Zinc oxide nanoparticles: A promising nanomaterial for biomedical applications. Drug Discov. Today 2017, 22, 1825-1834. [CrossRef]

13. Joe, A.; Park, S.-H.; Shim, K.-D.; Kim, D.-J.; Jhee, K.-H.; Lee, H.-W.; Heo, C.-H.; Kim, H.-M.; Jang, E.-S. Antibacterial mechanism of $\mathrm{ZnO}$ nanoparticles under dark conditions. J. Ind. Eng. Chem. 2017, 45, 430-439. [CrossRef]

14. Zimniewska, M.; Goslinska-Kuzniarek, O. Evaluation of Antibacterial Activity of Flax Fibres Against the Staphylococcus aureus Bacteria Strain. Fibres Text. East. Eur. 2016, 24, 120-125. [CrossRef] 
15. Tian, Y.; Liu, X.; Zheng, X.; Wang, L. Antimicrobial Properties of Flax Fibers in the Enzyme Retting Process. Fibres Text. East. Eur. 2016, 24, 15-17. [CrossRef]

16. Khan, B.A.; Warner, P.; Wang, H. Antibacterial Properties of Hemp and Other Natural Fibre Plants: A Review. BioResources 2014, 9, 3642-3659. [CrossRef]

17. ASTM. ASTM E2149-01, Standard Test Method for Determining the Antimicrobial Activity of Immobilized Antimicrobial Agents under Dynamic Contact Conditions; ASTM International: West Conshohocken, PA, USA, 2001.

18. Klančnik, A.; Toplak, N.; Kovač, M.; Marquis, H.; Jeršek, B. Quantification of Listeria monocytogenes cells with digital PCR and their biofilm cells with real-time PCR. J. Microbiol. Methods 2015, 118, 37-41. [CrossRef]

19. ISO. ISO 11721-1:2001, Textiles-Determination of Resistance of Cellulose-Containing Textiles to Micro-Organisms-Soil Burial Test-Part 1: Assessment of Rot-Retardant Finishing; ISO: Geneva, Switzerland, 2001.

20. ISO. ISO 1172-1:2003, Textiles-Determination of the Resistance of Cellulose-Containing Textiles to Micro-Organisms-Soil Burial Test-Part 2: Identification of Long-Term Resistance of a Rot Retardant Finish; ISO: Geneva, Switzerland, 2003.

21. Karthik, S.; Siva, P.; Balu, K.S.; Suriyaprabha, R.; Rajendran, V.; Maaza, M. Acalypha indica- mediated green synthesis of $\mathrm{ZnO}$ nanostructures under differential thermal treatment: Effect on textile coating, hydrophobicity, UV resistance, and antibacterial activity. Adv. Powder Technol. 2017, 28, 3184-3194. [CrossRef]

22. Fouda, A.; Hassan, S.E.-D.; Salem, S.S.; Shaheen, T.I. In-Vitro cytotoxicity, antibacterial, and UV protection properties of the biosynthesized Zinc oxide nanoparticles for medical textile applications. Microb. Pathog. 2018, 125, 252-261. [CrossRef]

23. Souza, D.A.R.; Gusatti, M.; Ternus, R.Z.; Fiori, M.A.; Riella, H.G. In Situ Growth of ZnO Nanostructures on Cotton Fabric by Solochemical Process for Antibacterial Purposes. J. Nanomater. 2018, 2018, 9082191. [CrossRef]

24. Fiedot, M.; Maliszewska, I.; Rac-Rumijowska, O.; Suchorska-Woźniak, P.; Lewińska, A.; Teterycz, H. The Relationship between the Mechanism of Zinc Oxide Crystallization and Its Antimicrobial Properties for the Surface Modification of Surgical Meshes. Materials 2017, 10, 353. [CrossRef]

25. Fiedot-Toboła, M.; Ciesielska, M.; Maliszewska, I.; Rac-Rumijowska, O.; Suchorska-Woźniak, P.; Teterycz, H.; Bryjak, M. Deposition of Zinc Oxide on Different Polymer Textiles and Their Antibacterial Properties. Materials 2018, 11, 707. [CrossRef]

26. Paduraru, A.; Ghitulica, C.; Trusca, R.; Surdu, V.A.; Neacsu, I.A.; Holban, A.M.; Birca, A.C.; Iordache, F.; Vasile, B.S. Antimicrobial Wound Dressings as Potential Materials for Skin Tissue Regeneration. Materials 2019, 12, 1859. [CrossRef]

27. Shao, D.F.; Wei, Q.F. Microwave-Assisted Rapid Preparation of Nano-ZnO/Ag Composite Functionalized Polyester Nonwoven Membrane for Improving Its UV Shielding and Antibacterial Properties. Materials 2018, 11, 1412. [CrossRef]

28. Gao, D.; Li, Y.; Lyu, B.; Lyu, L.; Chen, S.; Ma, J. Construction of durable antibacterial and anti-mildew cotton fabric based on P(DMDAAC-AGE)/Ag/ZnO composites. Carbohydr. Polym. 2019, 204, 161-169. [CrossRef]

29. Primc, G.; Tomšič, B.; Vesel, A.; Mozetič, M.; Ražić, S.E.; Gorjanc, M. Biodegradability of oxygen-plasma treated cellulose textile functionalized with $\mathrm{ZnO}$ nanoparticles as antibacterial treatment. J. Phys. D Appl. Phys. 2016, 49, 324002. [CrossRef]

30. Klemenčič, D.; Simončič, B.; Tomšič, B.; Orel, B. Biodegradation of silver functionalised cellulose fibres. Carbohydr. Polym. 2010, 80, 426-435. [CrossRef]

31. Tomšič, B.; Simončič, B.; Orel, B.; Vilčnik, A.; Spreizer, H. Biodegradability of cellulose fabric modified by imidazolidinone. Carbohydr. Polym. 2007, 69, 478-488. [CrossRef]

32. Socrates, G. Infrared and Raman Characteristic Group Frequencies; John Wiley \& Sons: New York, NY, USA, 2001; p. 347.

(C) 2019 by the authors. Licensee MDPI, Basel, Switzerland. This article is an open access article distributed under the terms and conditions of the Creative Commons Attribution (CC BY) license (http://creativecommons.org/licenses/by/4.0/). 